\title{
Componente teórico para un modelo tutorial en práctica docente en estudiantes para profesor de matemáticas: reflexión desde la experiencia en contextos de aprender a enseñar \\ Theoretical component for a tutorial model in educational practice in students for professor of mathematics: reflection from the experience in contexts to learn to teach
}

Fernando Guerrero R.

\begin{abstract}
Resumen
El modelo de devolución planteado por Brousseau (1998) se explora en las prácticas docentes de los Estudiantes para Profesores de Matemáticas ${ }^{2}$ para reflexionar sobre y en la acción docente en el aula de matemáticas. Para ello en el espacio de formación de la clase de práctica docente se pretende generar conocimiento en la acción desde la tutoría que lleva a cabo el profesor de práctica sobre el diseño y planeación, gestión y evaluación de una secuencia didáctica en torno a la comprensión de conceptos matemáticos en la Educación Básica. La tutoría del profesor de práctica se asume como un practicum reflexivo a través de la resolución de problemas del profesor.
\end{abstract}

El profesor de práctica genera condiciones para que en el espacio de formación de la tutoría, los EPPs aprendan a tomar decisiones sobre el proceso instructivo, este conocimiento práctico les sirve para apoyar su propio juicio sobre aprender a enseñar.

\begin{abstract}
The model of return raised by Brousseau (1998) is explored in the educational practices of the Students for Professors of mathematics 3to reflect on and in the educational action in the classroom of mathematics. For it in the space of formation of the class of educational practice it is tried to generate knowledge in the action from the position of a guardian that carries out the professor of practice on the design and planning, manage-
\end{abstract}

1 Pregrado Universitario Universidad de Nariño Licenciatura en Matemáticas, especialización Fundación Alberto Merani Para El Desarrollo de la Inteligencia. Instituto Alberto Merani. Universidad Distrital Francisco José de Caldas. E-mail: nfguerreror@udistrital. edu.co

2 En adelante se usa la sigla EPPS para referir a los Estudiantes para profesor de matemáticas de la Educación básica. Esta experiencia tiene lugar en el curso de práctica intermedia de octavo semestre del proyecto curricular de Licenciatura en Educación básica con énfasis en Matemáticas de la Universidad Distrital Francisco José de Caldas.

3 In ahead abbreviation EPPS is used to refer to the Students for professor of mathematics of the basic Education. This experience takes place in the course of intermediate practice of eighth semester of the curricular project of Degree in basic Education with emphasis in Mathematics of the Distrital University Francisco Jose de Caldas. 
ment and evaluation of a didactic sequence around the understanding of mathematical concepts in the basic Education. The position of a guardian of the practice professor is assumed like a reflective practicumthrough the resolution of problems of the professor.

The practice professor generates conditions so that in the space of formation of the position of a guardian, the epps learn to make decisions on the instructive process, this practical knowledge serves to them to support his own judgment on learning to teach.

\section{Palabras clave:}

Práctica docente, aprender a enseñar, Conocimiento Didáctico de Contenido (CDC), conocimiento base para la enseñanza, prácticum reflexivo, Teoría de las Situaciones Didácticas (TSD), conocimiento práctico, razonamiento pedagógico, Modelo Teórico Local (MTL)

\section{Key words:}

Educational Practice, To Learn To Teach, Didactic Knowledge of Content (CDC), Knowledge Bases for Education, Reflective Prácticum, Theory of the Didactic Situations (TSD), Practical Knowledge, Pedagogical Reasoning, Local Theoretical Model (MTL)

\section{Modelos teóricos locales}

El presente trabajo parte del concepto de Modelo Teórico Local propuesto por Eugenio Filloy hace algunos años, y desarrollado recientemente por Puig y Rojano. Para Puig (2007) los estudios de este estilo parten de una toma de partido teórica por no utilizar teorías generales de la enseñanza, el aprendizaje o la comunicación; por el contrario, se trata de elaborar modelos teóricos locales para dar cuenta de los procesos que se desarrollan cuando se enseña en el sistema educativo unos contenidos matemáticos concretos a unos alumnos concretos, y sólo se pretende que esos modelos sean adecuados para los fenómenos observados. Ahora bien, a la vez que se dice que el ámbito de validez de los modelos no se afirma que vaya más allá de los fenómenos observados, también se afirma que la descripción de los fenómenos que el modelo procura es profunda, compleja y minuciosa, y, para ello, es preciso que los modelos teóricos locales contemplen cuatro componentes: el componente de competencia del Modelo Teórico Local o, de forma abreviada, el Modelo de competencia - formal, si es el caso-; el componente de actuación del Modelo Teórico Local o Modelo de actuación - que, si hacemos la hipótesis de que las actuaciones las podemos describir en términos de procesos cognitivos, podemos denominar Modelo de cognición-; el componente de enseñanza del Modelo Teórico Local o Modelo de Enseñanza; $y$, finalmente, el componente de comunicación del Modelo Teórico Local o Modelo de comunicación.

\section{Puig (2007, p.12), recalca que:}

El carácter local viene dado por el hecho de que el modelo se elabora para dar cuenta de los fenómenos que se producen en los procesos de enseñanza y aprendizaje de unos contenidos matemáticos concretos a unos alumnos concretos y sólo se pretende que el modelo sea adecuado para los fenómenos observados. El carácter de modelo viene dado, entre otras cosas, por el hecho que no se hace la afirmación fuerte que las cosas son tal y como las caracteriza el modelo, sino sólo que, si las cosas fueran como las caracteriza el modelo, los fenómenos se producirían como se han descrito. El modelo tiene pues carácter descriptivo, explicativo y predictivo, pero no excluye que los mismos fenómenos puedan describirse, explicarse y predecirse de otra manera (mediante otro modelo). En esto se diferencia la pretensión de la elaboración del modelo de la pretensión que suele acompañar la elaboración de una teoría, que implica la exclusión de cualquier otra teoría que se avance para explicar los mismos hechos, a la que se combatirá como errónea.

Según este autor los MTL se elaboran para dar cuenta de fenómenos que se producen en situaciones de enseñanza y aprendizaje, las cuales se entienden en 
este contexto como situaciones de comunicación y de producción de sentido.

\section{Resolución de problemas del profesor o el modelo de actuación}

Según Guerrero, et al. $(2000,2005)$ constituye la resolución de problemas un aspecto fundamental de la práctica docente del profesor ya que indagando las concepciones y creencias que ha desarrollado sobre ella, puede dar cuenta de qué tipo de gestión curricular privilegia, en torno a lo declarado por él en su planeación y diseño de actividades, si es consistente con la gestión en el aula, cómo da cuenta de los aprendizajes alcanzados por los estudiantes a partir de la evaluación. De esta manera, la reflexión en la práctica antes, durante y después de la acción docente está mediada por la teoría didáctica como herramienta en el análisis de los procesos de enseñanza y aprendizaje (Guerrero, et al. 2005. p.3)

\section{Contextos de aprender a enseñar}

A partir de las investigaciones sobre la formación de los profesores sobre el pensamiento del profesor en la formación inicial algunos autores como Linares (1998), Blanco (1998), Florés (1999), Fonema (1995) se proponen el estudio del conocimiento profesional a partir de lo que estos autores han denominado los contextos de "aprender a enseñar", tomando en cuenta el estudio de distintos factores o variables de entrada como una manera de organizar tareas didácticas. En estudios recientes sobre prácticas en últimos años como se referencia a Marcelo (2009), considera unos principios o preceptos que implicaría considerar estos contextos.

Siguiendo a Marcelo (2009), entonces los preceptos hacen visible que durante la formación inicial de los profesores:

Se viene asumiendo una idea de que aprender a enseñar es un proceso activo y constructivo, que el aprendizaje está situado en contextos y culturas, se construye socialmente a través de la interacción de los individuos, en el que el futuro docente aprende y juega un papel importante en el proceso de aprendizaje de tal forma que es un constructor activo de conocimiento que da sentido al mundo interpretando las experiencias a través de sus conocimientos previos. Por otra parte se asume que el aprendizaje ocurre a través de la confrontación y transformación de los supuestos asumidos para resolver o reenfocar situaciones problemáticas y que el aprendizaje es un asunto que dura toda la vida y puede ser iniciado por el propio sujeto que aprende" (Marcelo citando a Flores, 2005, p.24).

Según este autor, estos preceptos se explicitan en los contextos de aprender a enseñar en comportamientos culturales y sociales en la formación inicial de los profesores tanto en los formadores como en los estudiantes para profesor, que se resumen en los siguientes aspectos:

- La formación del profesorado es inevitablemente insuficiente y no puede preparar a los profesores para toda su larga carrera. Esto nos sugiere que la formación del profesorado debe centrarse en cómo aprender de la experiencia y cómo construir conocimiento profesional.

- Aprender sobre la enseñanza requiere una visión del conocimiento como una materia por construir en lugar de como contenidos ya creados.

- Aprender a enseñar requiere un cambio de énfasis desde el currículo hacia los alumnos: un aspecto importante es que los profesores en formación deben tener oportunidades para acceder a pensamientos y acciones de los docentes de forma que les iluminen no sólo las acciones de enseñanza sino también los sentimientos y las razones que justifican una práctica docente. Ello requiere crear oportunidades para comprender lo que implica la planificación de la enseñanza, el desarrollo de la enseñanza, y reflexionar sobre ella. (Korthagen et al. 2006. p. 1029).

- Aprender a enseñar es un proceso que se construye a través de la investigación del profesor en formación. Este principio descansa en la idea de que los profesores en formación pueden investigar sobre su propia práctica. 
Los profesores en formación son futuros profesionales que son capaces de dirigir su propio desarrollo profesional investigando sobre su propia enseñanza.

- Aprender a enseñar requiere trabajar con otros compañeros.

- Es importante que los profesores aprendan que la colaboración con otros compañeros forma parte de la profesión docente para romper el aislamiento característico de la enseñanza.

- Aprender a enseñar requiere relaciones significativas entre la escuela, la universidad y los profesores en formación. Los formadores de profesores deberían mantener una relación próxima con las escuelas y con la profesión docente.

- El proceso de aprender a enseñar se mejora cuando los enfoques de enseñanza y aprendizaje promovidos en el programa de formación son modelados por los formadores de profesores de su propia práctica.

Así para algunos miembros de la comunidad de educadores matemáticos aprender a enseñar está vinculado con la apuesta a la formación práctica, en el sentido de iniciar al estudiante en reconocer el aprendizaje en el aula como una experiencia enriquecedora tanto para los niños y jóvenes como para el mismo profesor en formación, además del modo como este aprendizaje está matizado por los ecosistemas conceptuales de los que participa el estudiante y el profesor - niveles en la noosfera educativa, microsistemas, endosistemas o mesosistemas-. Es decir, como se ve afectado directa o indirectamente por las políticas educativas ministeriales, por los proyectos educativos institucionales o por las decisiones que toma el profesor durante el proceso de enseñanza y aprendizaje de unos determinados conceptos matemáticos.

\section{Conocimiento Didáctico de contenido (CDC) o Modelo de enseñanza}

Como afirma Marcelo (2009. p. 28):

El conocimiento didáctico del contenido, como línea de investigación representa la confluencia de esfuerzos de investigadores didácticos con investigadores de materias específicas preocupados por la formación del profesorado. El conocimiento didáctico del contenido nos dirige a un debate en relación con la forma de organización, de representación, del conocimiento a través de analogías y metáforas. Plantea la necesidad de que los profesores en formación adquieran un conocimiento experto del contenido a enseñar, para que puedan desarrollar una enseñanza que propicie la comprensión de los alumnos.

Esto quiere decir que en los programas de formación inicial de profesores se debe prestar atención al conocimiento que deben desarrollar sobre cómo se aprende a conocer la materia que enseña. Por ejemplo, dada la formación generalista que poseen los profesores de primaria, los futuros profesores deberían conocer con experticia el uso de materiales didácticos, de los centros de interés y teoría sobre cognición e instrucción matemática en la primaria. Todo esto para romper con la idea que "enseñar matemáticas en primaria reviste menos complejidad que en la secundaria". Así como "que la matemática en la educación primaria es más sencillo enseñar y por lo tanto aprender". Es decir, que existe un isomorfismo entre enseñar y aprender.

Shulman (1992) citado por Marcelo (2009), en la ponencia que presentó en el Congreso sobre las Didácticas Específicas en la Formación del Profesorado celebrado en Santiago, manifestaba la necesidad de que los profesores construyeran puentes entre el significado del contenido curricular y la construcción de ese significado por parte de los alumnos. Afirma este prestigioso investigador que:

Los profesores llevan a cabo esta hazaña de honestidad intelectual mediante una comprensión profunda, flexible y abierta del contenido; comprendiendo las dificultades más probables que tendrán los alumnos con estas ideas...; comprendiendo las variaciones de los métodos y modelos de enseñanza para ayudar a los alumnos en su construcción del conocimiento; y estando abierto a revisar sus objetivos, planes y procedimientos en la medida en 
que se desarrolla la interacción con los estudiantes. Este tipo de comprensión no es exclusivamente técnica, ni solamente reflexiva. No es sólo el conocimiento del contenido, ni el dominio genérico de métodos de enseñanza. Es una mezcla de todo lo anterior, y es principalmente pedagógico (Shulman, 1992. p. 12).

El conocimiento didáctico del contenido es un tipo de conocimiento que los profesores desarrollan acerca de la forma como comprenden los alumnos un determinado contenido. Incluye la formas de representar el contenido para hacerlo comprensible a los alumnos, las posibles dificultades que se pueden encontrar los alumnos cuando aprenden los contenidos (Borko \& Putnam, 1996). De acuerdo con Magnusson, Krajcik \& Borko (2003), el conocimiento didáctico del contenido incluye el conocimiento de temas concretos, problemas o aspectos por representar de un contenido.

¿Cómo podemos ayudar a los profesores a construir un conocimiento didáctico del contenido en el que se integre adecuadamente el conocimiento que ya poseen sobre la materia que enseñan con el conocimiento didáctico, el conocimiento de los alumnos, así como del currículum? Me parece que una buena vía de acceso a este conocimiento es a través del diseño de secuencias de aprendizaje por parte de los profesores en formación. Basándonos en Koper y Oliver:

Un diseño de aprendizaje se define como una aplicación de un modelo pedagógico para la consecución de un objetivo de aprendizaje concreto, para un determinado grupo de alumnos y para un contexto específico o un dominio determinado de conocimiento. El diseño de aprendizaje especifica procesos de enseñanza-aprendizaje.

De forma más concreta, específica bajo qué condiciones profesores y alumnos deben llevar a cabo las actividades que permitan a los alumnos alcanzar los objetivos de aprendizaje deseados. Un diseño de aprendizaje se puede referir a recursos físicos (objetos de aprendizaje y servicios de aprendizaje) que se necesitan durante el proceso de enseñanza-aprendizaje (Koper \& Olivier, 2004. p. 98).

Cabe resaltar que la pretensión de diseñar un aprendizaje rebasa la posibilidad de conocer en qué condiciones un estudiante acepta el compromiso de construir su propio conocimiento y también que como sostiene Gross (2008) "haya diseño o no el estudiante aprende o no". Es decir, que la conducta es solo una reacción al diseño del ambiente de aprendizaje y no su consecuencia.

Según este autor, citado por Marcelo (2009. p. 28): "Así, en todo proceso de diseño del aprendizaje se incluyen al menos los siguientes Componentes:

- Persona: una persona adopta un rol en el proceso de enseñanza-aprendizaje, normalmente un alumno o un profesor. Cada uno de estos roles se pueden especificar en sub-roles. En una simulación, diferentes alumnos pueden jugar diferentes roles.

- En este rol, esta persona trabaja para conseguir algunos resultados llevando a cabo actividades de aprendizaje y de apoyo, dentro de un ambiente.

Los ambientes consisten en objetos de aprendizaje y servicios apropiados para utilizar durante la realización de las actividades.

- El método determina la coordinación de roles, actividades y ambientes asociados que permiten a los alumnos conseguir las metas de aprendizaje.

- Las actividades se ensamblan en estructuras de actividades. Una estructura de actividad agrega un conjunto de actividades relacionadas dentro de una estructura simple. Una estructura de actividad puede modelar una secuencia o selección de actividades. En una secuencia, la persona debe completar diferentes actividades en el orden proporcionado. En la selección la persona seleccionaba un determinado número de actividades a partir de un conjunto dado.

- Los ambientes contienen los recursos y referencias necesarias para llevar a cabo una acti- 
vidad o conjunto de actividades. Un ambiente contiene:

- - Objetos de aprendizaje: cualquier entidad que se utiliza para aprender: páginas web, artículos, libros, bases de datos, software.

- Servicios de aprendizaje: por ejemplo, comunicaciones, control, colaboración.

- Subambientes.

- Condiciones: un método puede contener condiciones. Las reglas «si... entonces que» se pueden utilizar para personalizar el diseño del aprendizaje.

- Propiedades: se refieren a los criterios que aportan información en relación con las personas o papeles a lo largo de la unidad de aprendizaje. En nuestro grupo de investigación venimos trabajando en la identificación y descripción de secuencias de aprendizaje por parte de docentes que puedan ilustrar procesos de aprendizaje de los alumnos y que puedan ser aplicadas a diferentes contenidos de enseñanza. ¿Qué le pedimos a los docentes? A través de una entrevista le solicitamos que nos describan una secuencia completa (que puede abarcar un tema, un módulo o un curso completo) haciendo hincapié en que no sólo deben describir qué hacen ellos, sino formalidad e informalidad en el proceso de aprender a enseñar Principalmente ¿cuáles son las tareas de aprendizaje que los alumnos deben realizar? Estas tareas de aprendizaje son variadas: individuales, grupales, de asimilación, comunicación, aplicación, producción, etc. Y las tareas de aprendizaje se asocian con los apoyos que reciben los alumnos o el docente para su desarrollo, así como los recursos que se emplean.

Lo que aquí se llama diseño de aprendizaje se debe entender mejor como una micro ingeniería didáctica en el sentido de Artigue (1995), como una sucesión articulada y coherente de un conjunto de actividades con fines de enseñanza en un tiempo determinado.

El análisis de la práctica a través de la revisión y ejemplificación de secuencias de aprendizaje pue- de ayudar a construir la identidad profesional docente que necesitamos.

Una identidad profesional comprometida con un aprendizaje de calidad para los alumnos. Una identidad que ayude a superar la creencia fuertemente asentada en muchos candidatos a profesores de secundaria de que para enseñar basta con saber la materia que se enseña.

La relación Universidad-Institución educativa: el lugar de las prácticas docentes de los EPP

La relación universidad-escuela está marcada por la tensión entre las prácticas docentes innovadoras y las tradicionales, en el sentido que apuntan en distintas direcciones. $\mathrm{Al}$ respecto sostiene Marcelo (1994): “(...) la tendencia a conservar y mantener lo que está comprobado que funciona, es mayor que la capacidad de aceptación de las incertidumbres e inseguridades que cualquier cambio planificado comporta." (Marcelo, 1994. p.5)

La relación didáctica profesor, estudiante y saber en el entorno de la tutoría

Para potenciar el aprendizaje sobre la enseñanza y la adquisición y desarrollo del conocimiento didáctico de contenido el modelo de enseñanza del MTL en el entorno de la tutoría se fundamenta en los conceptos de estructura, apoyo y dirección.

Estructura: marco estable y consistente que permite reflexionar sobre las acciones de manera coherente (Atkinson, 2002).

Apoyo: es el interés activo que se reduce a interés positivo (Grolnik y Ryan, 1989)

Dirección: es la medida en la que está presente el “apoyo a la autonomía”. (Atkinson, 2002)

El propósito de poner en relación en la tutoría estos tres conceptos es la de favorecer la en los profesores en formación - estudiantes para profesor- la intuición en la toma de decisiones, para la adopción de juicios propios sobre la enseñanza de las nociones matemáticas. 
Entorno formativo para la tutoría (Atkinson, 2002)

El modelo de tutoría se organiza con estos conceptos mediante un esquema que sugiere Atkinson (2002) como un continuo de acciones de apoyo del formador sobre los procesos de aprender a enseñar del profesor en formación.

\section{Continúo de apoyo}

Poco apoyo $\longrightarrow$ Mucho apoyo

Continúo de estructura

Poco apoyo $\longrightarrow \quad$ Mucho apoyo

Continúo de dirección

Poco apoyo $\longrightarrow \quad$ Mucho apoyo

Por ejemplo, como ya se menciono antes, el diseño de un aprendizaje o de una secuencia de actividades supone un continuo de acciones de apoyo, estructura y dirección para la selección con un criterio teórico de los problemas, de la selección de las variables didácticas, de los observables para la detección y explicación de los errores y dificultades de los estudiantes. En general, de lo que el profesor en formación debe poner en juego como conocimiento base para la enseñanza de las matemáticas en la educación primaria o secundaría.

Efectos en el entorno de la tutoría en los profesores en formación (EPPs) sobre la capacidad para confiar en los propios juicios (Atkinson, 2002)

Así como Brousseau (1986) define elementos de la modelización para explicar el proceso instructivo en el seno de un sistema didáctico, en particular, los efectos Jourdin, Topaze, deslizamiento metacognitivo, envejecimiento de las situaciones didácticas Atkinson (2002), describe acciones del formador en la tutoría para potenciar el aprendizaje para confiar en los juicios propios en los EPPs para la toma de decisiones para el diseño de un aprendizaje fundamentados en la interrelación entre los conceptos de apoyo, estructura y dirección.

\section{APOYO ABUNDANTE DIRECCIÓN ABUNDANTE}

El apoyo se manifiesta en los juicios del formador - la dependencia de las direcciones externas se refuerza - la capacidad para confiar en los juicios se deteriora.

\section{APOYO ABUNDANTE DIRECCIÓN ESCASA}

El apoyo se centra en los juicios del propio sujeto y en sus resultados - Disminuye la necesidad de dirección externa - la capacidad para confiar en los juicios se desarrolla

\section{APOYO ABUNDANTE ESTRUCTURA ESCASA}

Se apoyan indiscriminadamente acciones inconsistentes - se engendra una sensación de infalibilidad - confianza implícita e irreflexiva en los juicios

\section{APOYO ABUNDANTE ESTRUCTURA ABUNDANTE}

Se apoyan aquellos actos que encajan en el entorno-se refuerza la estructura, se reflexiona sobre los actos intuitivos y se adecuan-se promueve la confianza en los propios juicios.

\section{Modelo de comunicación o producción de sentido}

Los estudiantes para profesor reconocen en su reflexión sobre la acción que la tutoría en práctica docente se vuelve crucial porque toma decisiones sobre el diseño, la gestión y evaluación, en tanto, este conocimiento práctico le sirve para apoyar su propio juicio.

Afirman que la práctica docente es compleja pues se constituye como conocimiento en acción, pues en ella se involucran distintos factores como el conocimiento sobre el currículo de matemáticas, sobre los aprendices, sobre las nociones matemáticas y el proceso instructivo. Reconocen rupturas en la manera de entender la resolución de problemas como metodología de clase según las formas de intervenir en el aula, en el paso de la idea de actividad como contenido a problema matemático.

Sostienen que en la tutoría son importantes las manifestaciones conscientes o no de sus aversiones o preferencias por la resolución de problemas, en tanto, si el modelo tutorial propiciado por el formador se fundamenta sobre las ideas de ambiente favorable - organización de un formato adecuado para el diseño de la actividad matemática para el estudiante-, apoyo a la autonomía —entrega de la responsabilidad para la selección de las actividades 
e implementación desde un modelo de intervención cuyo esquema se vincule con la resolución de problemas - y cantidad de apoyo ofrecido - ideas del tutor para mejorar los diseños, sugerencias sobre cómo hacer una sistematización más acorde con los objetivos propuestos y el marco teórico considerado, entre otros- implica esto que se favorece el autoestima - seguridad y confianza en el juicio propio-y la intuición —creatividad en el razonamiento pedagógico suscitado con la acción-.

En la práctica docente del estudiante para profesor (EPP) su motivación está dominada porque el alumnado de la escuela vea que las matemáticas escolares sirven de instrumento de comunicación y modelización. Este hecho ha servido para que entiendan que el apoyo se centra en sus propios juicios y en sus resultados, en una disminución de la necesidad de dirección externa, aumentando su capacidad para confiar en los juicios que desarrolla. (Atkinson, 2001)

Por otra parte, los estudiantes para profesor señalan que el modelo de devolución de Brousseau (1998), les ha servido para indagar sobre sus propios procesos de pensamiento sobre el conocimiento que desarrollan sobre la materia que enseñan, sobre lo que es posible aprender en primaria y secundaria, sobre la concepción de práctica matemática y el desarrollo de competencias para la toma de decisiones sobre la construcción de conocimiento en el aula como una comunidad de aprendizaje.

El modelo de devolución planteado por Brousseau (1998) puede ser tomado como referente en las prácticas docentes de los EPP para reflexionar sobre/en la acción y generar conocimiento en la acción desde la tutoría como un practicum reflexivo a través de la resolución de problemas del profesor. A la vez que el EPP enseña al alumnado de secundaria y primaria, él aprende a tomar decisiones en la gestión en el aula.

\section{Modelo de competencia en el proceso de aprender a enseñar}

¿Qué sabe el profesor en formación sobre la materia a enseñar?
La didáctica de la matemática como herramienta de análisis y diseño de aprendizaje.

¿Cómo son las tramas conceptuales que desarrolla el profesor en formación en una práctica matemática necesaria en el proceso de aprender a enseñar?

\section{C.D.C}

Epistemología de la práctica

Razonamiento pedagógico y conocimiento práctico.

¿Cuáles son los elementos que constituyen teóricamente una práctica reflexiva en el estudiante para profesor en torno al diseño de aprendizaje?

Planeación y diseño

- Secuencia de actividades

- Preparación de clases

Guía del profesor

Elementos constitutivos

Guía del estudiante

Taller para el desarrollo de la temática

Gestión en el aula

- Variables didácticas

De la actividad

Organización en el aula

Del estudiante

Evaluación

- Indicadores de logro

Conceptuales, procedimentales

Niveles de complejidad

Practicum reflexivo (shon)

Taller en forma de asesoría al diseño presentado por el epp para la gestión en el aula

- Dialogo entre el estudiante para profesor (epp) y el tutor para mejorar el diseño

- Reflexión sobre comentarios previos al encuentro

REVISTA CIENTÍFICA / ENERO -DICIEMBRE DE 2009 / No. 11 / BOGOTÁ, D.C. 
Orientaciones sobre / en la acción implementada por el epp

- Como mejorar la intervención

Conocimiento en acción

- Decisiones que modifican el diseño previo

A partir de las manifestaciones de la devolución

Gestión de la resolución de problemas

Reflexión en la acción

- Con la intervención del profesor tutor a partir de los protocolos o vídeos de la gestión realizada por el epp

\section{Bibliografía}

Atkinson, T. y Claxton, G. (2002). El profesor intuitivo. Barcelona: Octaedro.

Brousseau, G. (1986). Métodos y fundamentos en Didáctica de las matemáticas. Acceso: 12 de Noviembre de 2009.

Guerrero, et al. (2000). Resolución de problemas del profesor de matemáticas. En: II Encuentro colombiano de Matemática Educativa, ASOCOLME.

Guerrero, F et al. (2005). La práctica docente a partir de los modelos DECA y Teoría de situaciones didácticas. En: RELME. (19), Montevideo.

Guerrero, F. (2009). Análisis de un modelo tutorial fundamentado en algunos conceptos de la TSD. En: VIII Congreso internacional en investigación en enseñanza de las ciencias. Barcelona.

Lurduy, O, et al. (2005). Funciones y roles del profesor de matemáticas: El caso de la función lineal. En: ASOCOLME (7)

Sanchez, N. et al. (2002). Experiencias de aula. En: Cuadernos de investigación No 5. La ruta de aprendizaje: el caso de la matemática. Bogotá: FPUD.

Sanchez, N. y Guerrero, F. (2000). A propósito del saber del profesor: una reflexión en tor- no al algoritmo de la suma. En: Revista EMA, No.5.

Sanchez, N. y Guerrero, F (2003). Renovación curricular: la práctica docente como eje articulador del conocimiento profesional. En: Red CEE. Lajeado, Brasil

Schon, D. (1992). La formación de profesionales reflexivos. Hacia un nuevo diseño de la enseñanza y aprendizaje en las profesiones. Barcelona: Paidos.

Schon, D. (1998). El profesional reflexivo. Cómo piensan los profesionales cuando actúan. Barcelona: Paidos.

Llinares, S. (1995). Conocimiento base para la enseñanza de las matemáticas. En: Santalo, L (1995). Enseñanza de la matemática en la Educación Intermedia. Madrid: Rialp.

Marcelo, C. (1992). Como conocen los profesores la materia que enseñan. Algunas contribuciones de la investigación sobre conocimiento didáctico del contenido. Ponencia presentada en el congreso "Las didácticas específicas en la formación del profesorado". Santiago, 6-10 de julio. Acceso: 12 de Noviembre de 2009.

Marcelo, C. (1994). Investigaciones sobre prácticas en los últimos años. Qué nos aportan para la mejora cualitativa de las prácticas. Ponencia presentada al III Symposium Internacional sobre Prácticas Escolares, Poio, Junio, 1994. Acceso: 12 de Julio de 2006. Disponible en :

Marcelo, C. (2009). Formalidad e informalidad en el proceso de aprender a enseñar. En Revista de Educación. (350). Septiembre-diciembre 2009. pp. 31-55. Acceso: 12 de Noviembre de 2009. Disponible en :

Puig, L. (2008). Sentido y elaboración del componente de competencia de los modelos teóricos locales en la investigación de la enseñanza y aprendizaje de contenidos matemáticos específicos. PNA, 2(3). pp. 87-107. Acceso: 22 de septiembre de 2009. 